\title{
Two-Stage Peroral Endoscopic Myotomy for Sigmoid-Type Achalasia
}

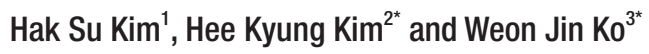 \\ ${ }^{1}$ Department of Gastroenterology and Hepatology, Incheon Sarang Hospital, Incheon, ${ }^{2}$ Department of Gastroenterology and Hepatology, \\ Cheonggu Sungsim Hospital, Seoul, ${ }^{3}$ Department of Gastroenterology, CHA Kumi Medical Center, Gumi, Korea
}

Peroral endoscopic myotomy (POEM) has been recently considered as the first treatment option for achalasia. The standard POEM procedures are often successful in most patients, but sometimes technical challenges are encountered. We report a new technique that is divided between two tunneling sites in the esophagus for sigmoid-type achalasia. A 40-year-old male patient with dysphagia for 10 years was diagnosed with a sigmoid-shaped esophagus at our hospital. We devised a two-stage myotomy technique to treat sigmoidtype achalasia. The myotomy was first performed in the upper part of the greater flexion area and then in the lower part of the flexion. We termed this method "two-stage POEM", which was successfully performed without any complications. This new POEM method can also be used to improve symptoms in patients with achalasia who have a structural deformity that may result in a high change of treatment failure. Clin Endosc 2020;53:232-235

Key Words: Esophageal achalasia; Esophageal motility disorders; Myotomy; Peroral endoscopic myotomy

\section{INTRODUCTION}

Achalasia is a neurodegenerative motility disorder that is caused by impaired relaxation of the lower esophageal sphincter (LES), increased LES tone, and absent peristalsis of the esophagus. ${ }^{1}$ Peroral endoscopic myotomy (POEM) is known to be a safe and effective approach for patients with esophageal achalasia compared to surgical treatment. Inoue et al. introduced POEM in 2009 as a non-surgical approach to treat a patient with esophageal achalasia in response to rapidly increasing less invasive treatment requirements. ${ }^{2,3}$ Currently, POEM is considered as the first treatment for achalasia, and the indications for POEM have been expanding to sigmoid-shaped achalasia.

Received: March 15, 2019 Revised: April 9, 2019

Accepted: April 16, 2019

Correspondence: Weon Jin Ko

Department of Gastroenterology, CHA Kumi Medical Center, CHA University, 12 sinsi-ro 10-gil, Gumi 39295, Korea

Tel: +82-54-450-9562, FAX: +82-303-0450-9885, E-mail: wisred@naver.com ORCID: https://orcid.org/0000-0002-3796-8539

*These authors contributed equally to this study.

(c) This is an Open Access article distributed under the terms of the Creative Commons Attribution Non-Commercial License (http://creativecommons.org/ licenses/by-nc/3.0) which permits unrestricted non-commercial use, distribution, and reproduction in any medium, provided the original work is properly cited.
The POEM procedure for sigmoid-shaped achalasia is more difficult than for other non-sigmoid-type achalasia. The sigmoid-shaped esophagus is considered to a highly advanced achalasia, in which the lumen of the esophagus is significantly rotated with dilatation. ${ }^{3}$ As such, advanced achalasia requires other treatment approaches. Here, we report a new technique that is divided between two tunneling sites in the esophagus with flexion, termed "two-stage POEM" for sigmoid-type achalasia.

\section{CASE REPORT}

A 40-year-old man visited our hospital with dysphagia that had gradually worsened over the 10 years prior to the visit. He had no known underlying disease or significant family history. On the first esophagogastroduodenoscopy at admission, his esophagus showed retention of fluid and food materials with severe dilatation. He had an Eckardt symptom score of 8. During a barium esophagography, a significant amount of contrast agent remained in the esophagus after 5 minutes and showed a typical bird beak appearance (Fig. 1A). In high-resolution manometry, the integrated relaxation pressure was 11 $\mathrm{mmHg}$, and the patient was subsequently diagnosed with type 
I achalasia with a sigmoid-shaped esophagus (Fig. 1B).

After the general anesthesia, intravenous antibiotics were administered before POEM. A 9.8-mm diameter high-definition esophagogastroscope (GIF-H260; Olympus, Tokyo, Japan) was used for the procedure. The mucosal incision was performed $33 \mathrm{~cm}$ from the upper incisors. As in the case of general POEM, myotomy of the inner circular muscle was made by using an IT knife and an F knife. First, a submu- cosal tunnel was made after a submucosal saline injection using an endoscopic knife in the upper part of the greater flexion area (Fig. 2A), and then myotomy was performed in this area (Fig. 2B). Subsequently, a second submucosal tunnel was made, and myotomy was performed again in the lower part of the flexion (Fig. 2C, D). Finally, endoclipping was used for closure. We termed this method "two-stage POEM", which was successfully performed without any complications
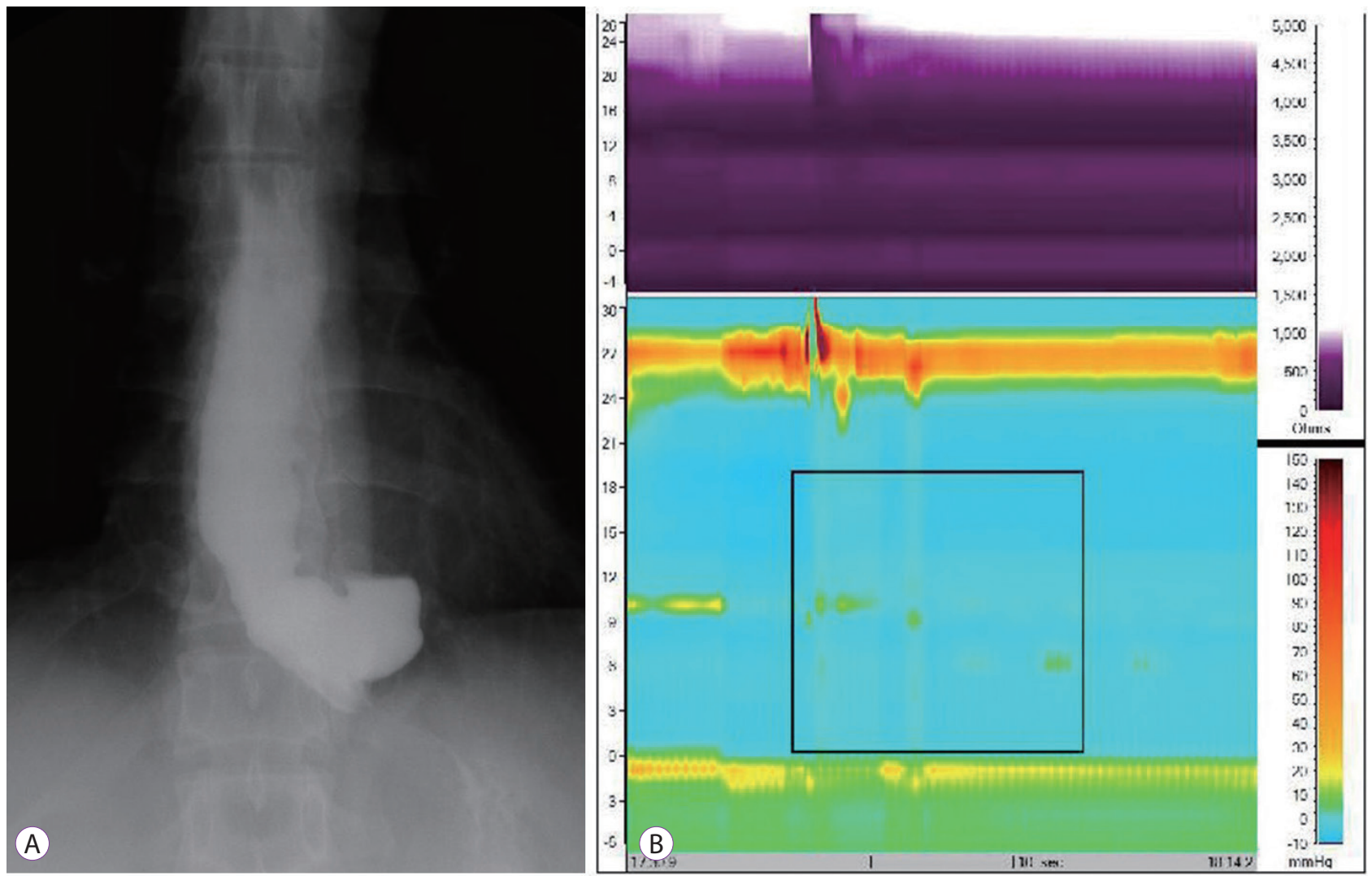

Fig. 1. (A) Preoperative barium esophagography showing the remaining contrast medium, which was about $13 \mathrm{~cm}$ in length with a bird beak appearance in the esophagus (sigmoid-shaped esophagus). (B) High-resolution manometry showing the absence of peristalsis and no pressurization within the esophageal body as well as high integrated relaxation pressure.
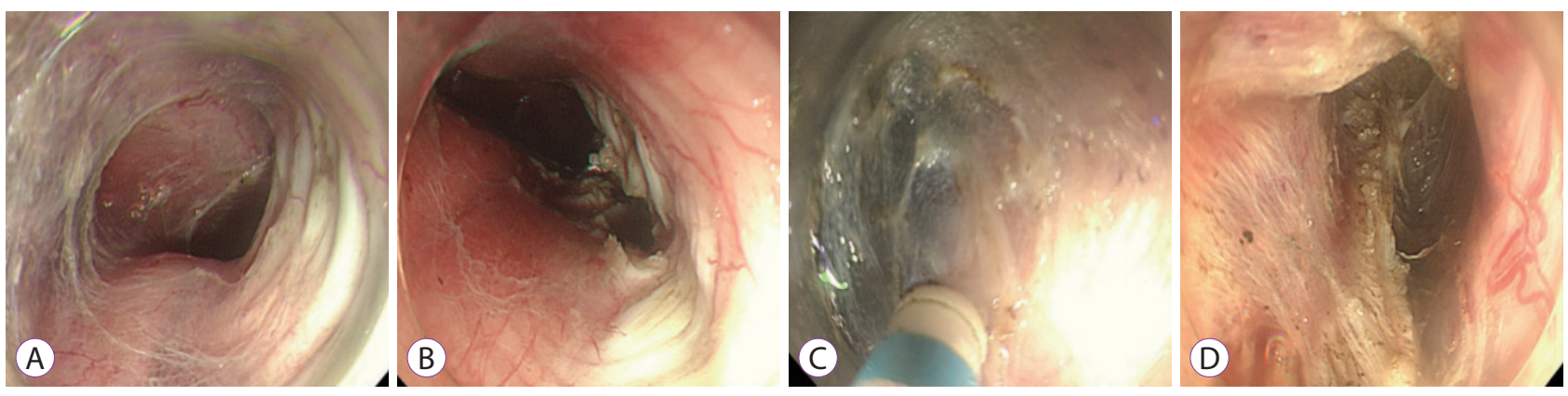

Fig. 2. The peroral endoscopic myotomy procedure. (A) The first submucosal tunnel was made after submucosal saline injection using an endoscopic knife at the upper part of the greater flexion area. (B) Myotomy was then performed at this area. (C) The second submucosal tunneling was performed at the lower part of the flexion. (D) A second myotomy was performed. 
(Supplementary video 1). The procedure time was $85 \mathrm{~min}$ and the myotomy length was $10 \mathrm{~cm}$ (esophagus, $8 \mathrm{~cm}$; stomach, $2 \mathrm{~cm}$ ).

Three days later, postoperative esophagography showed the rapid passage of barium from the esophagus into the stomach (Fig. 3). The following day, the patient was discharged from the hospital, and his Eckardt symptom score was reduced to 1 at 6 months after the treatment.

\section{DISCUSSION}

POEM has been demonstrated in several studies as an effective treatment for esophageal achalasia, but it remains controversial due to insufficient data from randomized controlled trials and a relatively short follow-up period. ${ }^{4}$ Recently, since the therapeutic range of POEM has expanded, a new

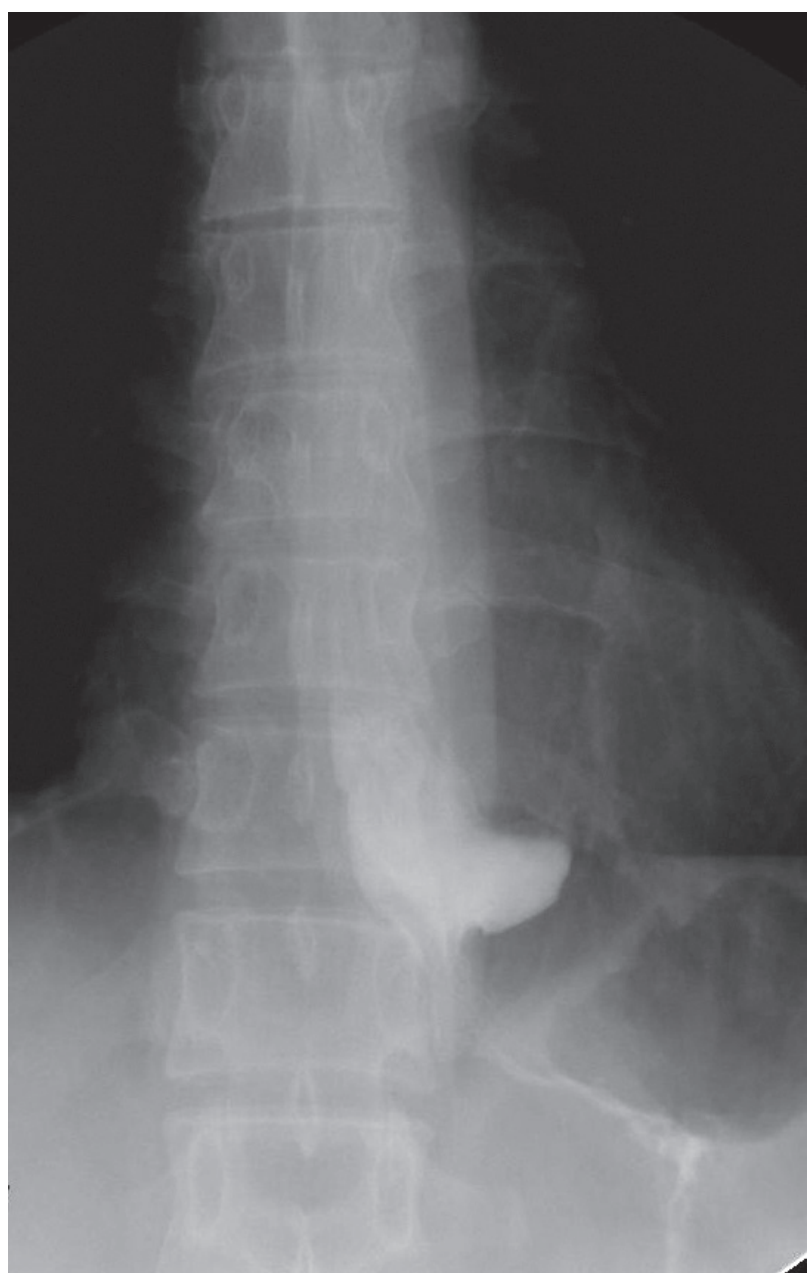

Fig. 3. After three days of the peroral endoscopic myotomy procedure, follow-up Barium esophagography showed barium contrast agents rapidly passing the esophagus. technological approach may present challenges in endoscopic treatments. Especially in cases of complicated esophageal achalasia, such as progressive sigmoid-shaped esophagus, a new technique is required because it is difficult to perform POEM in such cases. First, a submucosal tunnel is difficult to create without mucosal damage at the site of flexion. Secondly, the expanded and curved esophageal lumen may cause out of direction, and subsequent endoscopic dissection and detachment of the tissue surface is difficult.

Furthermore, patients with previous interventions show inflammation and fibrosis of the submucosal layer, which can interrupt submucosal tunneling. In a cohort study, submucosal fibrosis has been shown to be the most common cause of technical failure of POEM, and this often occurs when there is a history of interventions. ${ }^{5}$ They also found that any type of procedure could cause fibrosis, including general benign pneumatic dilation and botulinum toxin injection. A severe angulation also causes difficulties in establishing the mucosal tunneling direction. Lv et al. reported that a deep mucosal tunneling can help maintain the correct distal orientation of the mucosal tunnel by ensuring a direction perpendicular to the circular muscle fibers. ${ }^{3}$ Peroral endoscopic full-thickness myotomy has been attempted and reported to be effective for treatment. However, in this study, the overall reflux complication rate of POEM for sigmoid-shaped achalasia was $25.8 \%{ }^{3}$ Morphological changes in the esophagus can make endoscopic tunneling more difficult and time consuming. ${ }^{6,7}$ Liu et al. investigated the "open POEM", which was performed directly without a submucosal tunnel to treat sigmoid-type achalasia ${ }^{7}$ and was successfully performed without procedural side effects, resulting in a post-open POEM Eckardt score of 2 . These cases have even reported the technical efficacy and feasibility of POEM in patients with advanced achalasia; however, these cases are especially challenging and only experienced operators should perform these procedures.

As shown above, various new therapeutic approaches have been tried during POEM procedure for sigmoid-shaped achalasia. Here, we devised a two-stage myotomy technique. During the procedure, the mucosa is supported by the outside of the tunnel. Unexpected mucosectomy is one of the biggest problems when making the tunnel because patients with achalasia experience chronic inflammation of the mucosa. In particular, patients with sigmoid-type achalasia are more prone to flexion, resulting in more complications. If myotomy is performed first on the upper part of the flexion, the flexion site becomes wider, and subsequently, myotomy of the stomach can be performed more easily. In this patient, the procedure was completed successfully using this method. The procedure time was not long compared to other POEM procedures. However, a comparative study including more 
patients is needed to determine if this method is more useful and safe for sigmoid-type achalasia.

In conclusion, the symptoms of patients with achalasia who have structural deformities that can result in high chance of treatment failure can be improved using with 'two-stage POEM' method.

Conflicts of Interest

The authors have no conflicts of interest.

\section{Supplementary Material}

Video 1. Two-stage peroral endoscopic myotomy procedure for sigmoid-type achalasia. A submucosal tunnel was made at the upper part from the greater flexion, where a circular layer was cut in this area. Then, submucosal tunneling and full myotomy at the lower part was performed. Lastly, mucosal clipping was performed using endoscopic clips (https://doi. org/10.5946/ce.2019.067.v001).

\section{REFERENCES}

1. von Renteln D, Inoue $\mathrm{H}$, Minami $\mathrm{H}$, et al. Peroral endoscopic myotomy for the treatment of achalasia: a prospective single center study. Am J Gastroenterol 2012;107:411-417.

2. Inoue $\mathrm{H}$, Minami H, Satodate $\mathrm{H}$, Kudo S-E. First clinical experience of submucosal endoscopic esophageal myotomy for esophageal achalasia with no skin incision. Gastrointest Endosc 2009;69:AB122.

3. Lv L, Liu J, Tan Y, Liu D. Peroral endoscopic full-thickness myotomy for the treatment of sigmoid-type achalasia: outcomes with a minimum follow-up of 12 months. Eur J Gastroenterol Hepatol 2016;28:30-36.

4. Kim WH, Cho JY, Ko WJ, et al. Comparison of the outcomes of peroral endoscopic myotomy for achalasia according to manometric subtype. Gut Liver 2017;11:642-647.

5. Wu QN, Xu XY, Zhang XC, et al. Submucosal fibrosis in achalasia patients is a rare cause of aborted peroral endoscopic myotomy procedures. Endoscopy 2017;49:736-744.

6. Hu JW, Li QL, Zhou PH, et al. Peroral endoscopic myotomy for advanced achalasia with sigmoid-shaped esophagus: long-term outcomes from a prospective, single-center study. Surg Endosc 2015;29:2841-2850.

7. Liu W, Liu L, Chen HL, et al. Open peroral endoscopic myotomy for achalasia with sigmoid-shaped esophagus. Endoscopy 2017;49:E311-E312 